\title{
Improving an Anonymous and Provably Secure Authentication Protocol for a Mobile User
}

\author{
Jongho Moon, ${ }^{1}$ Youngsook Lee, ${ }^{2}$ Jiye Kim, ${ }^{3}$ and Dongho Won ${ }^{4}$ \\ ${ }^{1}$ Department of Electrical and Computer Engineering, Sungkyunkwan University, 2066 Seobu-ro, Jangan-gu, Suwon-si, \\ Gyeonggi-do 16419, Republic of Korea \\ ${ }^{2}$ Department of Cyber Security, Howon University, 64 Howondae 3-gil, Impi-myeon, Gunsan-si, \\ Jeonrabuk-do 54058, Republic of Korea \\ ${ }^{3}$ Department of Mobile Internet, Daelim University College, 29 Imgok-ro, Dongan-gu, Anyang-si, Gyeonggi-do 13916, Republic of Korea \\ ${ }^{4}$ Department of Computer Engineering, Sungkyunkwan University, 2066 Seobu-ro, Jangan-gu, Suwon-si, \\ Gyeonggi-do 16419, Republic of Korea \\ Correspondence should be addressed to Dongho Won; dhwon@security.re.kr
}

Received 4 May 2017; Accepted 16 August 2017; Published 27 September 2017

Academic Editor: Hongxin $\mathrm{Hu}$

Copyright (C) 2017 Jongho Moon et al. This is an open access article distributed under the Creative Commons Attribution License, which permits unrestricted use, distribution, and reproduction in any medium, provided the original work is properly cited.

\begin{abstract}
Recently many authentication protocols using an extended chaotic map were suggested for a mobile user. Many researchers demonstrated that authentication protocol needs to provide key agreement, mutual authentication, and user anonymity between mobile user and server and resilience to many possible attacks. In this paper, we cautiously analyzed chaotic-map-based authentication scheme and proved that it is still insecure to off-line identity guessing, user and server impersonation, and on-line identity guessing attacks. To address these vulnerabilities, we proposed an improved protocol based on an extended chaotic map and a fuzzy extractor. We proved the security of the proposed protocol using a random oracle and AVISPA (Automated Validation of Internet Security Protocols and Applications) tool. Furthermore, we present an informal security analysis to make sure that the improved protocol is invulnerable to possible attacks. The proposed protocol is also computationally efficient when compared to other previous protocols.
\end{abstract}

\section{Introduction}

Given recent developments in mobile telecommunications and the rapid spread of mobile devices, there is a growing importance of wireless and wired networking services that utilize bygone and current positional information from users carrying mobile devices with location tracking capabilities [1]. Remote user authentication schemes typically verify registered credentials using stored databases. Since Lamport [2] presented the first authentication scheme based on passwords in 1981, various remote user authentication schemes $[3,4]$ based on passwords have been proposed. However, since a server under a password-based remote user authentication protocol needs to store a verification table, which stores the password to determine the credentials of a remote user, the server arranges for extra storage for the verification table.
Furthermore, several studies have shown that passwordbased remote user authentication protocols are insecure against some attacks, including off-line password guessing or stolen smart card attacks [5-7]. The problem with passwordbased authentication scheme is that it can be easily stolen or lost and making it difficult to remember on a regular basis. For these reasons, many researchers have presented new remote user authentication protocols that use biometrics. A major characteristic of biometrics is it uniqueness. Other advantage is that it cannot be guessed or stolen. Biological characteristics have been used in numerous remote user authentication schemes [8-13].

To design a secure authentication scheme, some cryptographic algorithms are also used, such as an RSA cryptosystem [14,15], elliptic curve cryptography [16, 17], hash function $[18,19]$, and chaos-based cryptography [20-22]. 
Recently, many chaos-based authentication protocols have been suggested. Xiao et al. [23] first presented a user authentication protocol using a chaotic map and claimed that their protocol is useful and suitable for serviceable implementations. Unfortunately, many attacks were demonstrated by Han [31]. To overcome these vulnerabilities in [23], Han et al. [24] presented an enhanced user authentication protocol using chaos and asserted that their protocol resists all possible attacks. After that, Niu and Wang [32] proved that Han et al's protocol is vulnerable against an insider attack. Furthermore, Yoon [33] demonstrated that Niu and Wang's protocol does not resist a denial-of-service (DoS) attack. After that, Xue and Hong [34] proposed an improved authentication and key agreement protocol using a chaotic map to improve the security to some possible attacks. Unfortunately, Tan [35] found that Xue and Hong's protocol does not resist a manin-the-middle attack. Lee et al. [25] presented an improved chaotic map-based authentication protocol, and He et al. [29] proved that Lee et al.'s protocol does not resist DoS and insider attacks. To enhance the functionality and security, Lin [26] proposed a new authentication and key agreement protocol using a chaotic map and dynamic identity. Unfortunately, Islam et al. [27] found that Lin's protocol cannot resist wellknown attacks, and proposed an enhanced authentication protocol. However, we found that Islam et al.s protocol is still insecure against off-line identity guessing, impersonation, and on-line identity guessing attacks.

The remainder of this paper is organized as follows. We briefly introduce the Chebyshev chaotic maps, threat assumptions, and fuzzy extractor that we adopt in the proposed protocol in Section 2. In Sections 3 and 4, we, respectively, review and cryptanalyze Islam et al's protocol. In Section 5, we propose an improved authentication and key agreement protocol for a mobile user. In Section 6, we present a security analysis of the proposed protocol. Section 7 explains the functionality and performance analyses comparing the proposed protocol to previous protocols. The conclusions are presented in Section 8.

1.1. Our Contribution. To address the security vulnerabilities in Islam et al's authentication protocol and obtain the required performance, we propose a security-improved scheme. The primary contribution of this paper are described below.

(i) First, we prove that Islam et al.s protocol is still vulnerable to some attacks, and we show how an adversary can impersonate a legitimate user or server.

(ii) Second, we suggest an improved biometrics-based authentication and key agreement protocol on Islam et al's protocol. The improved protocol is designed to be secure to well-known attacks.

(iii) Third, we analyze that the proposed protocol has better robustness and a lower computational cost with a performance analysis.

\section{Preliminaries}

We briefly introduce the Chebyshev chaotic maps $[28,36]$, threat assumptions, and fuzzy extractor.
2.1. Chebyshev Chaotic Maps. The Chebyshev polynomial $T_{k}(v)$ is a $v$ polynomial of degree $k$.

Definition 1. Let $k$ be a whole number and $w$ be a real number from the round $[-1,1]$; the Chebyshev polynomial of degree $k$ is then defined as $T_{k}(v)=\cos (k \cdot \arccos (v))$.

Definition 2 (CMDLP). Given the two parameters $v, w \in Z_{n}^{*}$, the Chaotic Maps Discrete Logarithm Problem is whether integer $k$ can be found such that $w=T_{k}(v)$. The probability of $\mathscr{E}$ being able to address the CMDLP is defined as $\operatorname{Pr}\left[\mathscr{E}(v, w)=k: k \in Z_{n}^{*}, w=T_{k}(v) \bmod n\right]$.

Definition 3 (CMDHP). Given the three elements $v, T_{j}(v)$, and $T_{k}(v)$, the Chaotic Maps Diffie-Hellman Problem is whether $T_{j k}(v)$ can be computed such that $T_{j k}(v)=$ $T_{j}\left(T_{k}(v)\right)=T_{k}\left(T_{j}(v)\right)$.

2.2. Threat Assumptions. We introduce some threat model $[37,38]$ and consider constructing the threat assumptions described as follows:

(i) Adversary $\mathscr{E}$ can be both a user or server. Any registered mobile user can act as an adversary.

(ii) $\mathscr{E}$ can intercept all messages in a public channel, thereby capturing any message exchanged between a user or server.

(iii) $\mathscr{E}$ has the ability to modify, reroute, or delete the captured message.

(iv) Stored parameters can be extracted from the mobile device.

2.3. Fuzzy Extractor. In this subsection, we describe the basis for a biometric-based fuzzy extractor that converts biometric information data into a random value. Based on [39-41], the fuzzy extractor is operated through two procedures (Gen, Rep), demonstrated as

(i) $\mathrm{Gen}(\mathrm{BIO}) \rightarrow\langle\alpha, \beta\rangle$,

(ii) $\operatorname{Rep}\left(\mathrm{BIO}^{*}, \beta\right)=\alpha$ if $\mathrm{BIO}^{*}$ is reasonably close to BIO.

Gen is a probabilistic generation function for which the biometrics BIO returns an "extracted" string $\alpha \in\{0,1\}^{k}$ and auxiliary string $\beta \in\{0,1\}^{*}$, and Rep is a deterministic reproduction function that enables the recovery of $\alpha$ from $\beta$ and any vector $\mathrm{BIO}^{*}$ close to $\mathrm{BIO}$. Detailed information of the fuzzy extractor can be found in [42].

\section{Review of Islam et al.'s Protocol}

We review Islam et al.s protocol. Their protocol consists of registration, login, verification, and password change phases and uses an extended chaotic maps. The term $T_{k}(a)$ is the chaotic map computation that is calculated with respect to " $\bmod n$ " and $a \in(-\infty,+\infty)$. The notations of this paper are illustrated in the Notations. 


\subsection{Registration Phase}

(i) User $U_{i}$ selects the identity $\mathrm{ID}_{i}$ and password $\mathrm{PW}_{i}$ and inputs these values into the mobile devices $\mathrm{MD}_{i}$. $\mathrm{MD}_{i}$ then chooses a random number $t$, calculates $W_{i}=\mathrm{PW}_{i} \oplus t$, and sends $\left\langle\mathrm{ID}_{i}, W_{i}\right\rangle$ to server $S$ over an insecure channel.

(ii) Upon receiving $\left\langle\mathrm{ID}_{i}, W_{i}\right\rangle$, server $S$ computes $H_{i}=$ $h\left(s, \mathrm{ID}_{i}\right)$ and $n_{i}=h\left(W_{i}, \mathrm{ID}_{i}\right) \oplus\left(H_{i}, T_{s}\left(H_{i}\right)\right)$ and sends $\left\langle n_{i}\right\rangle$ to user $U_{i}$ by using a secure channel.

(iii) Upon receiving $\left\langle n_{i}\right\rangle, \mathrm{MD}_{i}$ retrieves $N_{i}=n_{i} \oplus h\left(W_{i}\right.$, $\left.\mathrm{ID}_{i}\right) \oplus h\left(\mathrm{ID}_{i}, \mathrm{PW}_{i}\right),\left(H_{i}, T_{s}\left(H_{i}\right)\right)=N_{i} \oplus h\left(\mathrm{ID}_{i}, \mathrm{PW}_{i}\right)$, and $X_{i}=h\left(h\left(\mathrm{ID}_{i}, \mathrm{PW}_{i}\right) \|\left(H_{i} \| T_{s}\left(H_{i}\right)\right)\right.$ and stores $\left\langle N_{i}, X_{i}\right\rangle$ into $\mathrm{MD}_{i}$.

\subsection{Login Phase}

(i) User $U_{i}$ enters $\mathrm{ID}_{i}$ and $\mathrm{PW}_{i}$ into $\mathrm{MD}_{i}$.

(ii) $\mathrm{MD}_{i}$ computes $\left(H_{i} \| T_{s}\left(H_{i}\right)\right)=N_{i} \oplus h\left(\mathrm{ID}_{i}, \mathrm{PW}_{i}\right)$ and $X_{i}^{\prime}=h\left(h\left(\mathrm{ID}_{i}, \mathrm{PW}_{i}\right) \|\left(H_{i} \| T_{s}\left(H_{i}\right)\right) . \mathrm{MD}_{i}\right.$ then checks whether $X_{i}^{\prime}$ is equal to $X_{i}$. If this holds, $\mathrm{MD}_{i}$ executes the following stage; otherwise, $\mathrm{MD}_{i}$ rejects the login request.

(iii) $\mathrm{MD}_{i}$ chooses a random number $k$ and then computes $Z_{i}=T_{k}\left(T_{s}\left(H_{i}\right)\right)$ and $\mathrm{CID}_{i}=\mathrm{ID}_{i} \oplus\left(H_{i}\left\|T_{1}\right\| Z_{i}\right)$, where $C_{i}=T_{k}\left(H_{i}\right), R_{i}=H_{i} \oplus Z_{i}, V_{i}=h\left(\mathrm{CID}_{i}\right.$, $\left.Z_{i}, H_{i}, R_{i}, T_{1}\right)$, and $T_{1}$ is the current timestamp. $\mathrm{MD}_{i}$ sends $\left\langle\mathrm{CID}_{i}, C_{i}, V_{i}, R_{i}, T_{1}\right\rangle$ to server $S$ by using a public channel.

\subsection{Verification Phase}

(i) When receiving the request message $\left\langle\mathrm{CID}_{i}, C_{i}\right.$, $\left.V_{i}, R_{i}, T_{1}\right\rangle$ from user $U_{i}$, server $S$ verifies freshness of timestamp $T_{1}$ and terminates the session if $\left(T_{2}-T_{1}\right) \leq$ $\Delta T$ is false; otherwise, server $S$ continues the next stage.

(ii) $S$ computes $Z_{i}=T_{s}\left(C_{i}\right), H_{i}=R_{i} \oplus Z_{i}, \mathrm{ID}_{i}=\mathrm{CID}_{i} \oplus$ $\left(H_{i}\left\|T_{1}\right\| Z_{i}\right)$, and $V_{i}^{\prime}=h\left(\mathrm{CID}_{i}, Z_{i}, H_{i}, R_{i}, T_{1}\right) . S$ then rejects the session if $V_{i}^{\prime} \neq V_{i}$; otherwise, server $S$ continues the following stage.

(iii) $S$ randomly chooses a number $l$ and computes the session key $\lambda=h\left(H_{i}, T_{1}, T_{2}, T_{l}\left(C_{i}\right)\right)$, and $V_{s}=h\left(\lambda, H_{i}\right.$, $\left.T_{1}, T_{2}\right)$. $S$ then sends the response messages $\left\langle V_{s}, T_{2}, T_{l}\left(H_{i}\right)\right\rangle$ over an insecure channel.

(iv) After receiving the response message $\left\langle V_{s}, T_{2}, T_{l}\left(H_{i}\right)\right\rangle$ from server $S$ at time $T_{3}, \mathrm{MD}_{i}$ checks the freshness of $T_{2}$ and terminates the session if $\left(T_{3}-T_{2}\right) \leq$ $\Delta T$ is false; otherwise, $\mathrm{MD}_{i} \mathrm{e}$ then computes $\lambda=$ $h\left(H_{i}, T_{1}, T_{2}, T_{k}\left(T_{l}\left(H_{i}\right)\right)\right)$, and $V_{s}^{\prime}=h\left(\lambda, H_{i}, T_{1}, T_{2}\right)$. $\mathrm{MD}_{i}$ next checks whether $V_{s}^{\prime} \stackrel{?}{=} V_{s}$. If this holds, $\mathrm{MD}_{i}$ accepts $\lambda$ as the session key and authenticates server $S$; otherwise, $\mathrm{MD}_{i}$ rejects the session.

\subsection{Password Change Phase}

(i) User $U_{i}$ inputs $\mathrm{ID}_{i}$ and $\mathrm{PW}_{i}$ into the mobile device $\mathrm{MD}_{i}$

(ii) $\mathrm{MD}_{i}$ computes $\left(H_{i} \| T_{s}\left(H_{i}\right)\right)=N_{i} \oplus h\left(\mathrm{ID}_{i}, \mathrm{PW}_{i}\right)$ and $X_{i}^{\prime}=h\left(h\left(\mathrm{ID}_{i}, \mathrm{PW}_{i}\right) \|\left(H_{i} \| T_{s}\left(H_{i}\right)\right)\right) . \mathrm{MD}_{i}$ then checks whether $X_{i}^{\prime}$ is the same to $X_{i}$. If this holds, the mobile device asks the new identity and password to $U_{i}$; otherwise, $\mathrm{MD}_{i}$ rejects the password change request.

(iii) $U_{i}$ inputs a new $\mathrm{ID}_{i}^{*}$ and $\mathrm{PW}_{i}^{*}$ into $\mathrm{MD}_{i} \cdot \mathrm{MD}_{i}$ then computes $N_{i}^{*}=N_{i} \oplus h\left(\mathrm{ID}_{i}, \mathrm{PW}_{i}\right) \oplus h\left(\mathrm{ID}_{i}^{*}, \mathrm{PW}_{i}^{*}\right)$ and $X_{i}^{*}=h\left(h\left(\mathrm{ID}_{i}^{*}, \mathrm{PW}_{i}^{*}\right) \|\left(H_{i} \| T_{s}\left(H_{i}\right)\right)\right)$ and replaces $\left\langle N_{i}, X_{i}\right\rangle$ by $\left\langle N_{i}^{*}, X_{i}^{*}\right\rangle$ into $\mathrm{MD}_{i}$.

\section{Cryptanalysis of Islam et al.'s Protocol}

We cryptanalyze the security problems in Islam et al's protocol [27]. Islam et al. analyzed the protocol by Lin et al. and improved it to support an improved security functionality. However, we found that Islam et al.s protocol was vulnerable to some possible attacks. These attacks are based on the threat assumptions that an adversary $\mathscr{E}$ was entirely monitored through the public channel connecting $U_{i}$ and $S$ in the login and verification phases and that $\mathscr{E}$ obtained the mobile device. Therefore, $\mathscr{E}$ can insert, modify, eavesdrop on, or delete any message transmitted over a public network. We now reveal further details of these problems.

4.1. Violation of the Identity. Let $\mathscr{E}$ be an active adversary who is a legitimate user and owns a mobile device to extract information $\left\langle N_{\mathscr{E}}, X_{\mathscr{E}}\right\rangle$ and suppose that an adversary $\mathscr{E}$ eavesdrops on the communication messages $\left\langle\mathrm{CID}_{i}, C_{i}\right.$, $\left.V_{i}, R_{i}, T_{1}, V_{s}, T_{2}, T_{l}\left(H_{i}\right)\right\rangle$ between user $U_{i}$ and server $S$. $\mathscr{E}$ can then easily obtain the identity of user $U_{i}$. The details are described as follows:

(i) Adversary $\mathscr{E}$ calculates $\left(H_{\mathscr{C}} \| T_{s}\left(H_{\mathscr{C}}\right)\right)=N_{\mathscr{E}} \oplus h\left(\mathrm{ID}_{\mathscr{E}}\right.$, $\left.\mathrm{PW}_{\mathscr{C}}\right)$.

(ii) Using [43], the adversary computes $s^{\prime}=$ $\left(\arccos \left(T_{s}\left(H_{\mathscr{C}}\right)\right)+2 k^{\prime} \pi\right) / \arccos \left(H_{\mathscr{C}}\right), \forall k \in Z$.

(iii) $\mathscr{E}$ can then compute $Z_{i}^{\prime}=T_{s^{\prime}}\left(C_{i}\right), H_{i}^{\prime}=R_{i} \oplus Z_{i}$, and $\mathrm{ID}_{i}=\mathrm{CID}_{i} \oplus\left(H_{i}^{\prime}\left\|T_{1}\right\| Z_{i}^{\prime}\right)$.

4.2. On-Line Identity Guessing and User Impersonation Attack. Let $\mathscr{E}$ be an active adversary who is a legitimate user and owns a mobile device to extract information $\left\langle N_{\mathscr{E}}, X_{\mathscr{E}}\right\rangle . \mathscr{E}$ can then easily guess the identity of any user $U_{i}$ and impersonate $U_{i}$ as follows.

(i) Adversary $\mathscr{E}$ computes $\left(H_{\mathscr{C}} \| T_{s}\left(H_{\mathscr{C}}\right)\right)=N_{\mathscr{E}} \oplus h\left(\mathrm{ID}_{\mathscr{E}}\right.$, $\left.\mathrm{PW}_{\mathscr{E}}\right)$.

(ii) $\mathscr{E}$ generates a random number $k$, computes $Z_{\mathscr{E}}=$ $T_{k}\left(T_{s}\left(H_{\mathscr{C}}\right)\right)$, guesses any identity $\mathrm{ID}_{i}$, and then computes $\mathrm{CID}_{i}=\mathrm{ID}_{i} \oplus\left(H_{\mathscr{E}}\left\|T_{1}\right\| Z_{\mathscr{E}}\right)$, where $C_{\mathscr{E}}=$ $T_{k}\left(H_{\mathscr{C}}\right), R_{\mathscr{C}}=H_{\mathscr{C}} \oplus Z_{\mathscr{C}}, V_{i}=h\left(\mathrm{CID}_{i}, Z_{\mathscr{C}}, H_{\mathscr{C}}, R_{\mathscr{E}}, T_{1}\right)$, and $T_{1}$ is the current time stamp. $\mathrm{MD}_{i}$ sends $\left\langle\mathrm{CID}_{i}, C_{\mathscr{E}}, V_{i}, R_{\mathscr{E}}, T_{1}\right\rangle$ to server $S$ over an insecure network. 


\begin{tabular}{|c|c|c|}
\hline User $U_{i}$ & & Server $S$ \\
\hline $\begin{array}{r}T_{r}\left(H_{i}\right)=v_{i} \oplus h\left(\mathrm{ID}_{i} \| \mathrm{DPW}_{i}\right) \\
V_{i}=h\left(\mathrm{ID}_{i} \| \mathrm{RPW}_{i}\right) \oplus T_{r}\left(H_{i}\right) \\
W_{i}=h\left(h\left(\mathrm{ID}_{i} \| \mathrm{RPW}_{i}\right) \| T_{r}\left(H_{i}\right)\right) \\
X_{i}^{\prime}=X_{i} \oplus t \\
\text { Delete }\left\langle t, v_{i}, X_{i}\right\rangle \\
\text { Store }\left\langle V_{i}, W_{i}, X_{i}^{\prime}\right\rangle\end{array}$ & $\left\langle\mathrm{ID}_{i}, \mathrm{DPW}_{i}=\mathrm{RPW} \oplus t\right\rangle$ & $\begin{array}{l}\text { Generate } y_{i} \\
H_{i}=h\left(s \| \mathrm{ID}_{i}\right) \\
v_{i}=h\left(\mathrm{ID}_{i} \| \mathrm{DPW}_{i}\right) \oplus T_{r}\left(H_{i}\right) \\
X_{i}=\left(y_{i} \| h\left(y_{i} \| s\right)\right) \oplus \mathrm{DPW}_{i}\end{array}$ \\
\hline
\end{tabular}

FIGURE 1: Registration phase of the proposed scheme.

(iii) Upon receiving the login request message $\left\langle\mathrm{CID}_{i}, C_{\mathscr{E}}\right.$, $\left.V_{i}, R_{\mathscr{E}}, T_{1}\right\rangle$ from the adversary $\mathscr{E}$, server $S$ verifies the freshness of the timestamp $T_{1}$ and terminates the session if $\left(T_{2}-T_{1}\right) \leq \Delta T$ is false; otherwise, server $S$ continues the next stage.

(iv) $S$ computes $Z_{\mathscr{E}}=T_{s}\left(C_{\mathscr{E}}\right), H_{\mathscr{E}}=R_{\mathscr{E}} \oplus Z_{\mathscr{E}}, \mathrm{ID}_{i}=$ $\mathrm{CID}_{i} \oplus\left(H_{\mathscr{E}}\left\|T_{1}\right\| Z_{\mathscr{E}}\right)$, and $V_{i}^{\prime}=h\left(\mathrm{CID}_{i}, Z_{\mathscr{E}}, H_{\mathscr{E}}\right.$, $\left.R_{\mathscr{E}}, T_{1}\right) . S$ then rejects the session if $V_{i}^{\prime} \neq V_{i}$; otherwise, server $S$ continues the following stage.

(v) $S$ randomly chooses a number $l$ and computes the session key $\lambda=h\left(H_{\mathscr{C}}, T_{1}, T_{2}, T_{l}(C)\right)$, and $V_{s}=h(\lambda$, $\left.H_{\mathscr{E}}, T_{1}, T_{2}\right)$. $S$ then sends the response messages $\left\langle V_{s}, T_{2}, T_{l}\left(H_{\mathscr{E}}\right)\right\rangle$ over an insecure channel.

(vi) After receiving the response messages $\left\langle V_{s}, T_{2}\right.$, $\left.T_{l}\left(H_{\mathscr{C}}\right)\right\rangle$ from server $S$ at time $T_{3}$, the mobile device checks the freshness of $T_{2}$ and terminates the session if $\left(T_{3}-T_{2}\right) \leq \Delta T$ is false; otherwise, $\mathrm{MD}_{i}$ then computes $\lambda=h\left(H_{\mathscr{C}}, T_{1}, T_{2}, T_{k}\left(T_{l}\left(H_{\mathscr{E}}\right)\right)\right)$. Finally, $\mathscr{E}$ and $S$ "successfully" conclude on the session key $\lambda$. However, server $S$ faultily decides that he/she is communicating with user $U_{i}$.

4.3. Server Impersonation Attack. Let $\mathscr{E}$ be an active adversary who is a legitimate user and owns a mobile device to extract information $\left\langle N_{\mathscr{E}}, X_{\mathscr{E}}\right\rangle . \mathscr{E}$ can then easily impersonate $S$ as follows.

(i) Adversary $\mathscr{E}$ computes $\left(H_{\mathscr{E}} \| T_{s}\left(H_{\mathscr{E}}\right)\right)=N_{\mathscr{E}} \oplus h\left(\operatorname{ID}_{\mathscr{E}}\right.$, $\left.\mathrm{PW}_{\mathscr{E}}\right)$.

(ii) Using [43], the adversary computes $s^{\prime}=$ $\left(\arccos \left(T_{s}\left(H_{\mathscr{E}}\right)\right)+2 k^{\prime} \pi\right) / \arccos \left(H_{\mathscr{E}}\right), \forall k \in Z$.

(iii) When receiving the login request message $\left\langle\mathrm{CID}_{i}\right.$, $\left.C_{i}, V_{i}, R_{i}, T_{1}\right\rangle$ from user $U_{i}, \mathscr{E}$ computes $Z_{i}^{\prime}=T_{s^{\prime}}\left(C_{i}\right)$ and $H_{i}=R_{i} \oplus Z_{i}^{\prime}$.

(iv) Adversary $\mathscr{E}$ randomly chooses a number $l$ and computes the session key $\lambda=h\left(H_{i}, T_{1}, T_{2}, T_{l}\left(C_{i}\right)\right)$, and $V_{s}=h\left(\lambda, H_{i}, T_{1}, T_{2}\right)$. The $\mathscr{E}$ then sends the response messages $\left\langle V_{s}, T_{2}, T_{l}\left(H_{i}\right)\right\rangle$ to user $U_{i}$ over an insecure channel.

(v) After receiving the response message $\left\langle V_{s}, T_{2}, T_{l}\left(H_{i}\right)\right\rangle$ from adversary $\mathscr{E}$ at time $T_{3}$, the mobile device checks the freshness of $T_{2}$ and terminates the session if $\left(T_{3}-\right.$ $\left.T_{2}\right) \leq \Delta T$ is false; otherwise, $\mathrm{MD}_{i}$ then computes $\lambda=$ $h\left(H_{i}, T_{1}, T_{2}, T_{k}\left(T_{l}\left(H_{i}\right)\right)\right)$, and $V_{s}^{\prime}=h\left(\lambda, H_{i}, T_{1}, T_{2}\right)$. The mobile device next checks whether $V_{s}^{\prime}=V_{s}$. If this holds, the mobile device accepts $\lambda$ as the session key. However, server $S$ faultily decides that he/she is communicating with $U_{i}$.

4.4. Violation of the Session Key. Assume that any adversary $\mathscr{E}$ eavesdrops on the communication messages $\left\langle\mathrm{CID}_{i}, C_{i}\right.$, $\left.V_{i}, R_{i}, T_{1}, V_{s}, T_{2}, T_{l}\left(H_{i}\right)\right\rangle$ between user $U_{i}$ and server $S$. $\mathscr{E}$ can then easily calculate the session key between $U_{i}$ and $S$.

(i) $\mathscr{E}$ calculates $\left(H_{\mathscr{E}} \| T_{s}\left(H_{\mathscr{E}}\right)\right)=N_{\mathscr{E}} \oplus h\left(\mathrm{ID}_{\mathscr{E}}, \mathrm{PW}_{\mathscr{E}}\right)$.

(ii) Using [43], the adversary computes $s^{\prime}=$ $\left(\arccos \left(T_{s}\left(H_{\mathscr{C}}\right)\right)+2 k^{\prime} \pi\right) / \arccos \left(H_{\mathscr{C}}\right), \forall k \in Z$.

(iii) $\mathscr{E}$ can compute $Z_{i}^{\prime}=T_{s^{\prime}}\left(C_{i}\right)$ and $H_{i}=R_{i} \oplus Z_{i}^{\prime}$.

(iv) Using [43], the adversary computes $k^{\prime}=\left(\arccos \left(C_{i}\right)+\right.$ $\left.2 k^{\prime} \pi\right) / \arccos \left(H_{i}\right), \forall k \in Z$.

(v) $\mathscr{E}$ can then compute the session key $\lambda=h\left(H_{i}, T_{1}\right.$, $\left.T_{2}, T_{k^{\prime}}\left(T_{l}\left(H_{i}\right)\right)\right)$.

\section{The Proposed Protocol}

We will propose an improved biometric-based authentication protocol using the fuzzy extractor. The proposed protocol is also two members, user $U_{i}$ and server $S$, and consists of four phases such as registration, login, verification, and password change. Figures 1 and 2 are the registration and login and verification phases of the proposed scheme. 


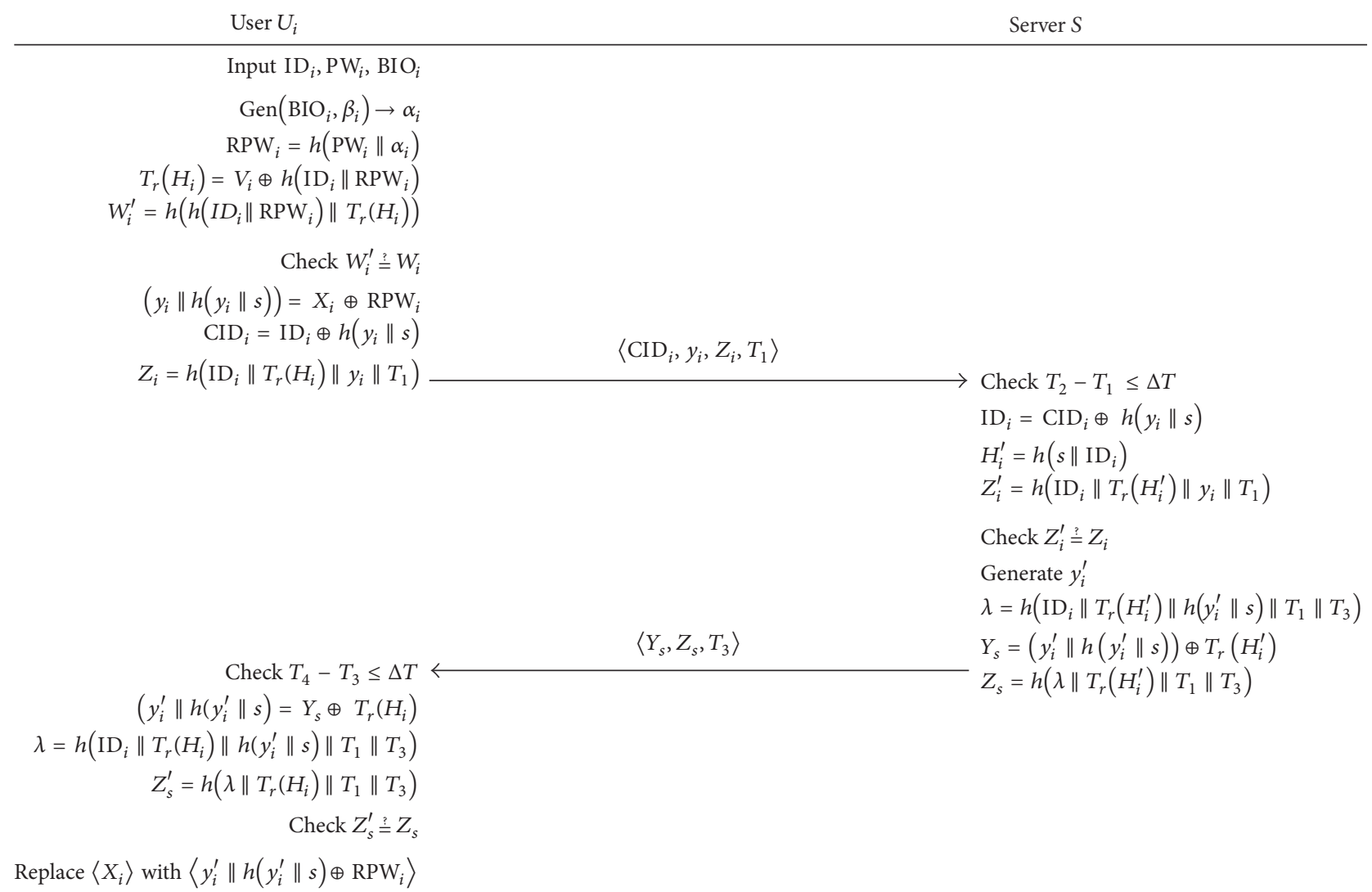

FIGURE 2: Login and verification phases of the proposed protocol.

\subsection{Registration Phase}

(i) $U_{i}$ gives one's biometrics $\mathrm{BIO}_{i}$ at the mobile device $\mathrm{MD}_{i}$. The $\mathrm{MD}_{i}$ then scans $\mathrm{BIO}_{i}$, pulls out two random strings $\left(\alpha_{i}, \beta_{i}\right)$ from the computation $\operatorname{Gen}\left(\mathrm{BIO}_{i}\right) \rightarrow$ $\left(\alpha_{i}, \beta_{i}\right)$, and stores $\beta_{i}$ in storage. $U_{i}$ enters the identity $\mathrm{ID}_{i}$ and password $\mathrm{PW}_{i}$, and $\mathrm{MD}_{i}$ then calculates $\mathrm{RPW}_{i}=h\left(\mathrm{PW}_{i} \| \alpha_{i}\right)$. Finally, $\mathrm{MD}_{i}$ generates a random number $t$, stores $t$ in the storage, and sends user registration request message $\left\langle\mathrm{ID}_{i}, \mathrm{DPW}_{i}=\mathrm{RPW}_{i} \oplus t\right\rangle$ to server $S$ by using a secure communication channel.

(ii) Upon receiving the request message for registration, $S$ randomly chooses a number $y_{i}$ and calculates $H_{i}=$ $h\left(s \| \mathrm{ID}_{i}\right), v_{i}=h\left(\mathrm{ID}_{i} \| \mathrm{DPW}_{i}\right) \oplus T_{r}\left(H_{i}\right)$, and $X_{i}=$ $\left(y_{i} \| h\left(y_{i} \| s\right)\right) \oplus \mathrm{DPW}_{i}$, where $r$ is a fixed random positive integer and $s$ is the master key of server $S$.

(iii) $S$ sends $\left\langle v_{i}, X_{i}\right\rangle$ to the $\mathrm{MD}_{i}$.

(iv) After receiving the registration response message $\left\langle v_{i}, X_{i}\right\rangle, \mathrm{MD}_{i}$ computes $T_{r}\left(H_{i}\right)=v_{i} \oplus h\left(\mathrm{ID}_{i} \| \mathrm{DPW}_{i}\right)$, $V_{i}=h\left(\mathrm{ID}_{i} \| \mathrm{RPW}_{i}\right) \oplus T_{r}\left(H_{i}\right), W_{i}=h\left(h\left(\mathrm{ID}_{i} \|\right.\right.$ $\left.\left.\mathrm{RPW}_{i}\right) \| T_{r}\left(H_{i}\right)\right)$, and $X_{i}^{\prime}=X_{i} \oplus t=\left(y_{\mathrm{i}} \| h\left(y_{i} \|\right.\right.$ $s)) \oplus \mathrm{RPW}_{i}$ and stores $\left\langle V_{i}, W_{i}, X_{i}^{\prime}\right\rangle$ into storage after deleting $t, v_{i}$, and $X_{i}$.

\subsection{Login Phase}

(i) $U_{i}$ enters $\mathrm{ID}_{i}$ and $\mathrm{PW}_{i}$ and gives $\mathrm{BIO}_{i}^{*}$ into the mobile device $\mathrm{MD}_{i}$.

(ii) $\mathrm{MD}_{i}$ scans $\mathrm{BIO}_{i}^{*}$ and recovers $\alpha_{i}$ from the computation $\operatorname{Rep}\left(\mathrm{BIO}_{i}^{*}, \beta_{i}\right) \rightarrow \alpha_{i}$.

(iii) $\mathrm{MD}_{i}$ then computes $\mathrm{RPW}_{i}=h\left(\mathrm{PW}_{i} \| \alpha_{i}\right), T_{r}\left(H_{i}\right)=$ $V_{i} \oplus h\left(\mathrm{ID}_{i} \| \mathrm{RPW}_{i}\right)$, and $W_{i}^{\prime}=h\left(h\left(\mathrm{ID}_{i} \| \mathrm{RPW}_{i}\right) \|\right.$ $T_{r}\left(H_{i}\right)$ ), and checks whether $W_{i}^{\prime}$ is the same to the stored $W_{i}$. If this holds, $\mathrm{MD}_{i}$ performs the next stage; otherwise, $\mathrm{MD}_{i}$ rejects the login request.

(iv) $\mathrm{MD}_{i}$ calculates $\left(y_{i} \| h\left(y_{i} \| s\right)\right)=X_{i} \oplus \mathrm{RPW}_{i}, \mathrm{CID}_{i}=$ $\mathrm{ID}_{i} \oplus h\left(y_{i} \| s\right)$, and $Z_{i}=h\left(\mathrm{ID}_{i}\left\|T_{r}\left(H_{i}\right)\right\| y_{i} \| T_{1}\right)$, where $T_{1}$ is the current timestamp.

(v) Finally, $\mathrm{MD}_{i}$ sends the request message $\left\langle\mathrm{CID}_{i}, y_{i}\right.$, $\left.Z_{i}, T_{1}\right\rangle$ for login to server $S$.

\subsection{Verification Phase}

(i) When receiving the request message $\left\langle\mathrm{CID}_{i}, y_{i}, Z_{i}, T_{1}\right\rangle$ from $\mathrm{MD}_{i}$, server $S$ checks whether $T_{2}-T_{1} \leq \Delta T$ is valid, where $\Delta T$ is the minimum acceptable time interval and $T_{2}$ is the actual arrival time of login request. If this holds, $S$ continues to proceed to the next stage; otherwise, $S$ rejects the request. 
(ii) $S$ then calculates $\mathrm{ID}_{i}=\mathrm{CID}_{i} \oplus h\left(y_{i} \| s\right), H_{i}=h(s \|$ $\left.\mathrm{ID}_{i}\right)$, and $Z_{i}^{\prime}=h\left(\mathrm{ID}_{i}\left\|T_{r}\left(H_{i}\right)\right\| y_{i} \| T_{1}\right)$ and checks whether $Z_{i}^{\prime}$ is the same to the received $Z_{i}$. If this holds, the $S$ continues to proceed to the next stage; otherwise, $S$ terminates this session.

(iii) $S$ randomly chooses a number $y_{i}^{\prime}$ and calculates the session key $\lambda=h\left(\mathrm{ID}_{i}\left\|T_{r}\left(H_{i}\right)\right\| h\left(y_{i}^{\prime} \| s\right)\left\|T_{1}\right\| T_{3}\right)$, $Y_{s}=\left(y_{i}^{\prime} \| h\left(y_{i}^{\prime} \| s\right)\right) \oplus T_{r}\left(H_{i}\right)$, and $Z_{s}=h\left(\lambda\left\|T_{r}\left(H_{i}\right)\right\|\right.$ $\left.T_{1} \| T_{3}\right) . S$ then sends the login response message $\left\langle Y_{s}, Z_{s}, T_{3}\right\rangle$ where $T_{3}$ is the current timestamp.

(iv) After receiving the response message $\left\langle Y_{s}, Z_{s}, T_{3}\right\rangle$ from server $S, \mathrm{MD}_{i}$ checks whether $T_{4}-T_{3} \leq \Delta T$ is valid, where $\Delta T$ is the minimum acceptable time interval and $T_{4}$ is the actual arrival time of response message. If this holds, $\mathrm{MD}_{i}$ continues to the next stage; otherwise, $\mathrm{MD}_{i}$ terminates this session.

(v) $\mathrm{MD}_{i}$ computes $y_{i}^{\prime} \| h\left(y_{i}^{\prime} \| s\right)=Y_{s} \oplus T_{r}\left(H_{i}\right)$ and the session key $\lambda=h\left(\operatorname{ID}_{i}\left\|T_{r}\left(H_{i}\right)\right\| h\left(y_{i}^{\prime} \| s\right)\left\|T_{1}\right\| T_{3}\right)$ and $Z_{s}^{\prime}=h\left(\lambda\left\|T_{r}\left(H_{i}\right)\right\| T_{1} \| T_{3}\right)$ and verifies whether $Z_{s}^{\prime}$ is the same to the received $Z_{s}$. If this holds, $\mathrm{MD}_{i}$ continues to the next stage; otherwise, $\mathrm{MD}_{i}$ terminates current session.

(vi) Finally, $\mathrm{MD}_{i}$ replaces $\left\langle X_{i}\right\rangle$ by $\left\langle\left(y_{i}^{\prime} \| h\left(y_{i}^{\prime} \| s\right)\right) \oplus\right.$ $\left.\mathrm{RPW}_{i}\right\rangle$ into storage.

\subsection{Password Change Phase}

(i) User $U_{i}$ inputs $\mathrm{ID}_{i}$ and $\mathrm{PW}_{i}$ and gives $\mathrm{BIO}_{i}^{*}$ into the mobile device $\mathrm{MD}_{i}$.

(ii) $\mathrm{MD}_{i}$ scans $\mathrm{BIO}_{i}^{*}$ and recovers $\alpha_{i}$ from the computation $\operatorname{Rep}\left(\mathrm{BIO}_{i}^{*}, \beta_{i}\right) \rightarrow \alpha_{i}$.

(iii) $\mathrm{MD}_{i}$ then computes $\mathrm{RPW}_{i}=h\left(\mathrm{PW}_{i} \| \alpha_{i}\right), T_{r}\left(H_{i}\right)=$ $V_{i} \oplus h\left(\mathrm{ID}_{i} \| \mathrm{RPW}_{i}\right)$, and $W_{i}^{\prime}=h\left(h\left(\mathrm{ID}_{i} \| \mathrm{RPW}_{i}\right) \|\right.$ $T_{r}\left(H_{i}\right)$ ) and checks whether $W_{i}^{\prime}$ is the same to the stored $W_{i}$. If this holds, $\mathrm{MD}_{i}$ performs the next stage; otherwise, $\mathrm{MD}_{i}$ rejects the password change request.

(iv) $U_{i}$ inputs a new password $\mathrm{PW}_{i}^{*}$ into $\mathrm{MD}_{i} \cdot \mathrm{MD}_{i}$ then computes $\mathrm{RPW}_{i}^{*}=h\left(\mathrm{PW}_{i}^{*} \| \alpha_{i}\right), V_{i}^{*}=h\left(\mathrm{ID}_{i} \|\right.$ $\left.\mathrm{RPW}_{i}^{*}\right) \oplus T_{r}\left(H_{i}\right), W_{i}^{*}=h\left(h\left(\mathrm{ID}_{i} \| \mathrm{RPW}_{i}^{*}\right) \| T_{r}\left(H_{i}\right)\right)$, and $X_{i}^{*}=X_{i} \oplus \mathrm{RPW}_{i} \oplus \mathrm{RPW}_{i}^{*}$.

(v) Finally, $\mathrm{MD}_{i}$ replaces $\left\langle V_{i}, W_{i}, X_{i}\right\rangle$ by $\left\langle V_{i}^{*}, W_{i}^{*}, X_{i}^{*}\right\rangle$ into storage.

\section{Security Analysis of the Improved Protocol}

The proposed protocol, which retains the advantages of Islam et al.s protocol, is demonstrated, and it can resist some possible attacks and supports all security properties. The analysis of the improved protocol was organized with the threat assumptions made in Preliminaries.

6.1. Formal Security Analysis. A random oracle-based formal analysis is demonstrated here, and its security is shown. First, the following hash function is defined [44]:
Definition 4. A collision-resistance and one-way hash function $h:\{0,1\}^{*} \rightarrow\{0,1\}^{k}$ receives an input as a binary string of arbitrary length $v \in\{0,1\}^{*}$, returns a binary string of fixed length $h(v) \in\{0,1\}^{k}$, and gratifies the following conditions:

(i) Given $w \in W$, it is computationally impracticable to find a $v \in V$ such that $w=h(v)$.

(ii) Given $v \in V$, it is computationally impracticable to find another $v^{\prime} \neq v \in V$, such that $h\left(v^{\prime}\right)=h(v)$.

(iii) It is computationally impracticable to find a pair $\left(v^{\prime}, v\right) \in V^{\prime} \times V$, with $v^{\prime} \neq v$, such that $h\left(v^{\prime}\right)=h(v)$.

Theorem 5. According to the assumptions if hash function $h(\cdot)$ similarly acts like an random oracle, then the improved protocol is clearly secure to an adversary $\mathscr{E}$ to protect sensitive information, including identity $I D_{i}$, semigroup property $T_{r}\left(H_{i}\right)$, common session key $\lambda$, and master secret keys.

Proof. Formal proof of the proposed protocol is similar in $[40,45]$, and it uses the oracle to construct $\mathscr{E}$, which will have the ability to extract $\operatorname{ID}_{i}, T_{r}\left(H_{i}\right), \lambda$, and $s$.

Reveal. Random oracle can extract input value $a$ from hash value $n=h(a)$ without failing. Adversary $\mathscr{E}$ now executes the experimental algorithm shown in Algorithm 1, EXP for the proposed scheme as BBSMK, for example. Let us then define the probability of success for $\operatorname{EXP}_{\mathrm{HASH}, \mathrm{A}}^{\mathrm{BBSMK}}$ as Success $_{\mathrm{HASH}, \mathrm{A}}^{\mathrm{BBSMK}}=\left|\operatorname{Pr}\left[\mathrm{EXP}_{\mathrm{HASH}, \mathrm{A}}^{\mathrm{BBSMK}}=1\right]-1\right|$, where $\operatorname{Pr}(\cdot)$ means the probability of $\operatorname{EXP}_{\mathrm{HASH}, \mathrm{A}}^{\mathrm{BBSMK}}$. The advantage function for this algorithm then defines $\operatorname{Adv}_{\mathrm{HASH}, \mathrm{A}}^{\mathrm{BBSMK}}\left(t, q_{R}\right)=\max _{\text {Success }}$, where $t$ and $q_{R}$ are the execution time and number of queries. We then discuss the algorithm in Algorithm 1 for $\mathscr{E}$. If $\mathscr{E}$ has the capability to address the problem of hash function given in Definition 4 , then he/she can immediately retrieve $\mathrm{ID}_{i}, T_{r}\left(H_{i}\right), \lambda$, and $s$. In that case, $\mathscr{E}$ will detect the complete connections between $U_{i}$ and $S$; however, the inversion of the input from a given hash result is not possible computationally; that is, $\operatorname{Adv}_{\mathrm{HASH}, \mathrm{A}}^{\mathrm{BBSMK}}(t) \leq \epsilon$, for all $\epsilon>0$. Thus, $\operatorname{Adv}_{\mathrm{HASH}, \mathrm{A}}^{\mathrm{BBSMK}}\left(t, q_{R}\right) \leq \epsilon$, since $\operatorname{Adv}_{\mathrm{HASH}, \mathrm{A}}^{\mathrm{BBSMK}}\left(t, q_{R}\right)$ depends on $\operatorname{Adv}_{\mathrm{HASH}, \mathrm{A}}^{\mathrm{BBSMK}}(t)$. In conclusion, there is no method for $\mathscr{E}$ to detect the complete connections between $U_{i}$ and $S$, and the proposed protocol is distinctly invulnerable to an adversary $\mathscr{E}$ to retrieve $\left(\mathrm{ID}_{i}, T_{r}\left(H_{i}\right), \lambda, s\right)$.

6.2. Simulation Result Using AVISPA. We perform to simulate the improved protocol for formal analysis using the widely accepted AVISPA. The main contribution of the simulation is to prove that the improved protocol is invulnerable to man-in-the-middle and replay attacks. AVISPA tool consists of four back-ends: (1) On-the-Fly Model Checker (OFMC); (2) Constraint-Logic-Based Attack Searcher; (3) SAT-Based Model Checker; and (4) Tree Automata Based on Automatic Approximations for the Analysis of Security Protocols. In the AVISPA, the protocol is implemented in High-Level Protocol Specification Language (HLPSL) [44], which is based on the roles: the basic roles for representing each entity role and composition roles for representing the scenarios of the basic 


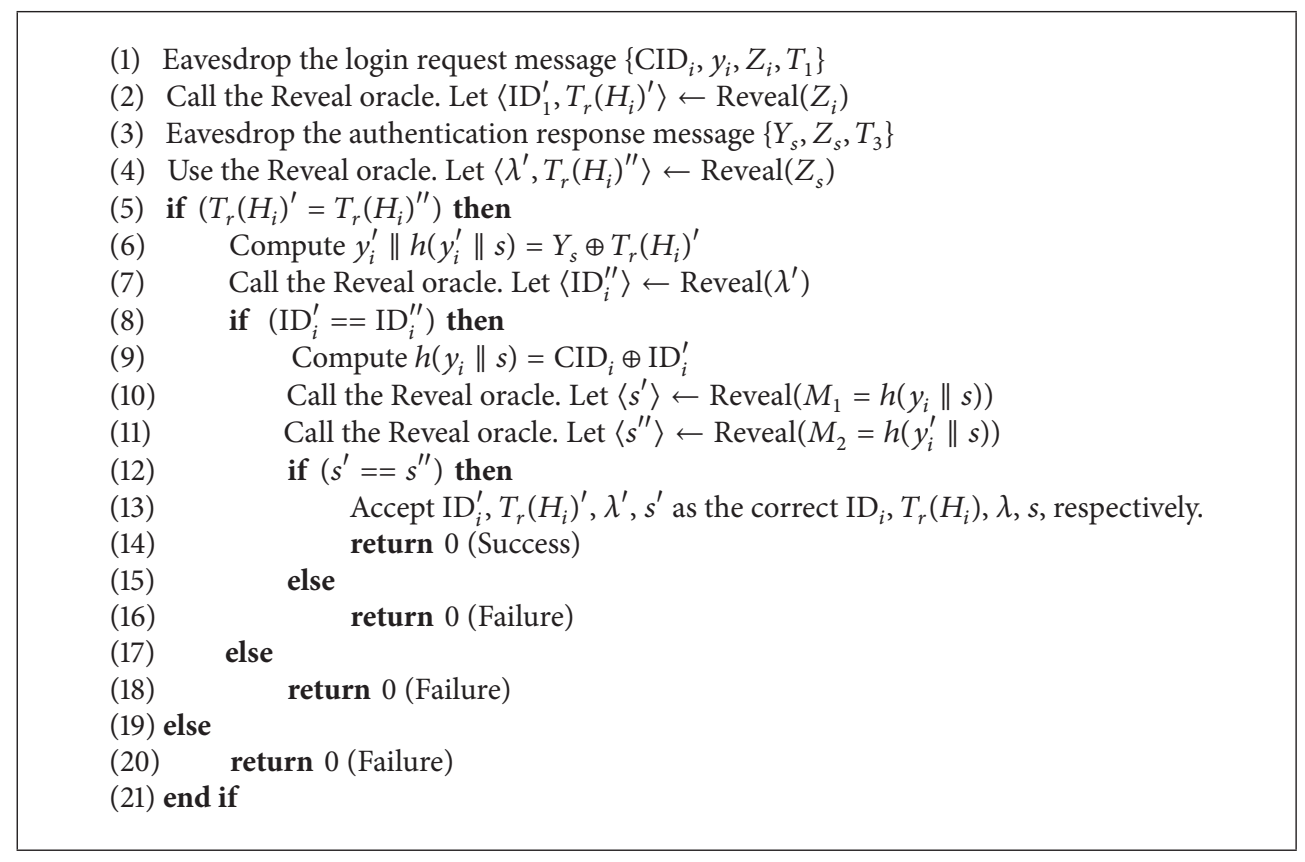

Algorithm 1: Algorithm EXP $\mathrm{HASH}, \mathrm{A}^{\mathrm{BBSMK}}$.

roles. The fundamental types available in the HLPSL are [46] as follows:

(i) agent: it means a primary name. The intruder always has the special identifier $i$.

(ii) symmetric key: it is the key using the symmetric-key cryptosystem.

(iii) text: the text values are applied for messages. They are often used as nonces.

(iv) nat: the nat is used for meaning the natural numbers in nonmessage contexts.

(v) const: it is the type for representing constants.

(vi) hash_func: the basic type hash_func expresses collision-resistance secure one-way hash functions.

The role of the initiator, user $U_{i}$, is shown in Algorithm 2. $U_{i}$ first receives the signal for starting and modifies its state variable from 0 to 1 . This state variable is retained by the variable state. Similar to user, the roles of server $S$ are implemented and shown in Algorithm 3. The specifications in HLPSL for the roles of environment, session, and goal are described in Algorithm 4. The result for the formal security verification of the improved protocol using OMFC is provided in Algorithm 5. It is clear that the improved protocol is invulnerable to passive and active attacks including the two attacks.

\subsection{Informal Security Analysis}

6.3.1. Mutual Authentication. Not only does the proposed scheme guarantee security as the other biometric-based schemes, but also $U_{i}$ and $S$ authenticate each other. $S$ authenticates $U_{i}$ by checking whether $Z_{i}$ is valid or not, because only a legitimate user can compute a valid $h\left(\mathrm{ID}_{i}\left\|T_{r}\left(H_{\mathrm{i}}\right)\right\| y_{i} \| T_{1}\right)$ using a chaotic map. $U_{i}$ then authenticates $S$ by checking $Z_{s}$, which only $S$ can compute using the long-term key $s$ and timestamp $T_{3}$.

6.3.2. User Anonymity. To compromise the anonymity of user $U_{i}$, adversary $\mathscr{E}$ must be able to compute $h\left(y_{i} \| s\right)$. The value $s$ is the master secret key of server $S$, and the random value $y_{i}$ changes every session. Thus, the login request message changes every session. Even if adversary $\mathscr{E}$ eavesdrops on the login request message of a user $U_{i}, \mathscr{E}$ does not know $\mathrm{ID}_{i}$. The proposed protocol provides user anonymity.

6.3.3. User Impersonation Attack. Suppose that an adversary $\mathscr{E}$ steals the mobile device $\mathrm{MD}_{i}$ of user $U_{i}$ and extracts the parameters $\left\{V_{i}, W_{i}, y_{i}, \beta_{i}, X_{i}\right\}$ from $\mathrm{MD}_{i}$. To make the login request message $\left\langle\mathrm{CID}_{i}, y_{i}, Z_{i}, T_{1}\right\rangle$, where $\mathrm{CID}_{i}=\mathrm{ID}_{i} \oplus h\left(y_{i} \|\right.$ $s)$ and $Z_{i}=h\left(\operatorname{ID}_{i}\left\|T_{r}\left(H_{i}\right)\right\| y_{i} \| T_{1}\right)$, the server's master key $s$ is needed. Without the master secret key $s$ from server $S$, $\mathscr{E}$ cannot compute $Z_{i}$. The proposed protocol can therefore resist a user impersonation attack.

6.3.4. Privileged Insider Attack. In the proposed protocol, user $U_{i}$ sends the login request message $\left\langle\mathrm{ID}_{i}, \mathrm{DPW}_{i}=\right.$ $\left.\mathrm{RPW}_{i} \oplus t\right\rangle$. Even if the privileged insider adversary $\mathscr{E}$ obtains these values $\left\langle\mathrm{ID}_{i}, \mathrm{DPW}_{i}=\mathrm{RPW}_{i} \oplus t\right\rangle, \mathscr{E}$ does not know $\mathrm{RPW}_{i}$ and cannot impersonate user $U_{i}$. The proposed protocol can therefore resist a privileged insider attack.

6.3.5. Lost Mobile Device Attack. Suppose that user $U_{i}$ 's mobile device $\mathrm{MD}_{i}$ has been stolen or lost and any adversary $\mathscr{E}$ obtains it. $\mathscr{E}$ then tries to login to server $S$ using $\mathrm{MD}_{i}$; however, $\mathscr{E}$ does not know the correct password $\mathrm{PW}_{i}$. To 
role user (Ui, AS: agent,

SKuas: symmetric_key,

$\mathrm{H}$, F: function,

SND, RCV: channel (dy))

played_by Ui def=

local State: nat,

IDi, PWi, BIOi, RPWi, DPWi, T, Ai: text,

Hi, Vi, VVi, R, S, Xi, Yi, Wi: text,

CIDi, Zi, T1, T3, SK, Y2, Ys, Zs: text

const as_ui_y2,

sc1, sc2, sc3, sc4: protocol_id

init State $:=0$

transition

(1) State $=0 \wedge R C V($ start $)=\mid>$

State' $:=1 \wedge \mathrm{T}^{\prime}:=\operatorname{new}()$

$\wedge$ RPWi' $:=\mathrm{H}($ PWi.Ai)

$\wedge$ DPWi' $:=\operatorname{xor}\left(\mathrm{RPWi}^{\prime}, \mathrm{T}^{\prime}\right)$

$\wedge \operatorname{secret}(\{\mathrm{PWi} . \mathrm{Ai}\}, \mathrm{scl}, \mathrm{Ui})$

$\wedge$ secret(IDi, sc2, $\{$ Ui,AS $\})$

$\wedge \mathrm{SND}(\{$ IDi.DPWi'\}_SKuas)

(2) State $=2 \wedge \operatorname{RCV}(\{\operatorname{xor}(H(I D i . x o r(H(P W i . A i), T ’)), F(R . H(S . I D i))) \cdot x o r((Y i \cdot H(Y i \cdot S))$, $\operatorname{xor}\left(\mathrm{H}\left(\mathrm{PWi}\right.\right.$.Ai), $\left.\left.\left.\mathrm{T}^{\prime}\right)\right)\right\}$ _SKuas $)=\mid>$

State' $:=4 \wedge \operatorname{secret}(\mathrm{R}, \mathrm{S}, \mathrm{sc} 3, \mathrm{AS})$

$\wedge \operatorname{secret}(\mathrm{F}(\mathrm{R} . \mathrm{H}(\mathrm{S} . \mathrm{IDi})), \mathrm{sc} 4, \mathrm{Ui}, \mathrm{AS})$

$\wedge$ VVi' := xor(H(IDi.H(PWi.Ai)), F(R.H(S.IDi)))

$\wedge \mathrm{Wi}^{\prime}:=\mathrm{H}(\mathrm{H}(\mathrm{IDi} . \mathrm{H}(\mathrm{PWi}$.Ai)).F(R.H(S.IDi)))

$\wedge \mathrm{Xi}^{\prime}:=\operatorname{xor}\left(\left(\mathrm{Yi}^{\prime} \mathrm{H}(\mathrm{Yi} \cdot \mathrm{S})\right), \mathrm{H}(\mathrm{PWi} . \mathrm{Ai})\right)$

$\wedge$ CIDi' := xor(IDi, H(Yi.S))

$\wedge \mathrm{Tl}^{\prime}:=$ new ()

$\wedge \mathrm{Zi}^{\prime}:=\mathrm{H}(\mathrm{IDi} . \mathrm{F}(\mathrm{R} . \mathrm{H}(\mathrm{S} . \mathrm{IDi}))$.Yi.T1')

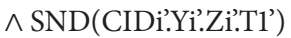

(3) State $=6 \wedge$ RCV (xor((Y2'.H(Y2'.S)),F(R.H(S.IDi))).H(SK.F(R.H(S.IDi)).T1'T3').T3') $=\mid>$

State' $:=8 \wedge \mathrm{SK}^{\prime}:=\mathrm{H}(\mathrm{IDi}$.F(R.H(S.IDi)).H(Y2'S).T1'T3')

$\wedge \mathrm{Xi}^{\prime}:=\operatorname{xor}\left(\left(\mathrm{Y} 2^{\prime} \cdot \mathrm{H}(\mathrm{Y} 2 \cdot \mathrm{S})\right), \mathrm{H}(\mathrm{PWi} . \mathrm{Ai})\right)$

$\wedge$ request(Ui, AS, as_ui_y2, Y2')

end role

Algorithm 2: Role specification for user $U_{i}$.

login to $S$, the biometrics $\mathrm{BIO}_{i}$ is also needed. The proposed protocol can therefore resist a lost mobile device attack.

6.3.6. Replay Attack. One of the best solutions to prevent replay attack is to use a timestamp technique. The proposed protocol also uses timestamps. Even if any adversary $\mathscr{E}$ eavesdrops on any user's login request message and sends it to the server $S$, the server $S$ checks the freshness of the timestamp and rejects the request. Furthermore, an adversary $E$ cannot compute $Z_{i}$ without $\mathrm{ID}_{i}$ and $y_{i}$. The proposed protocol can therefore resist a replay attack.

6.3.7. Off-Line Password Guessing Attack. To obtain a password of user $U_{i}$, the biometrics $\mathrm{BIO}_{i}$ is needed. Biometrics is uniquene and it cannot be guessed or stolen. The proposed protocol can therefore resist an off-line password guessing attack.

6.3.8. Stolen Verifier Attack. In the proposed protocol, a server $S$ does not store any information related to the user's identity or password. The proposed protocol can therefore resist a stolen verifier attack.

6.3.9. Session Key Forward Security. One important objective of any user authentication protocols is to constitute a session key between user $U_{i}$ and server $S$. The forward secrecy can protect previous and future session keys from adversary $\mathscr{E}$ if the master secret key of $S$ is exposed. Suppose that the master 


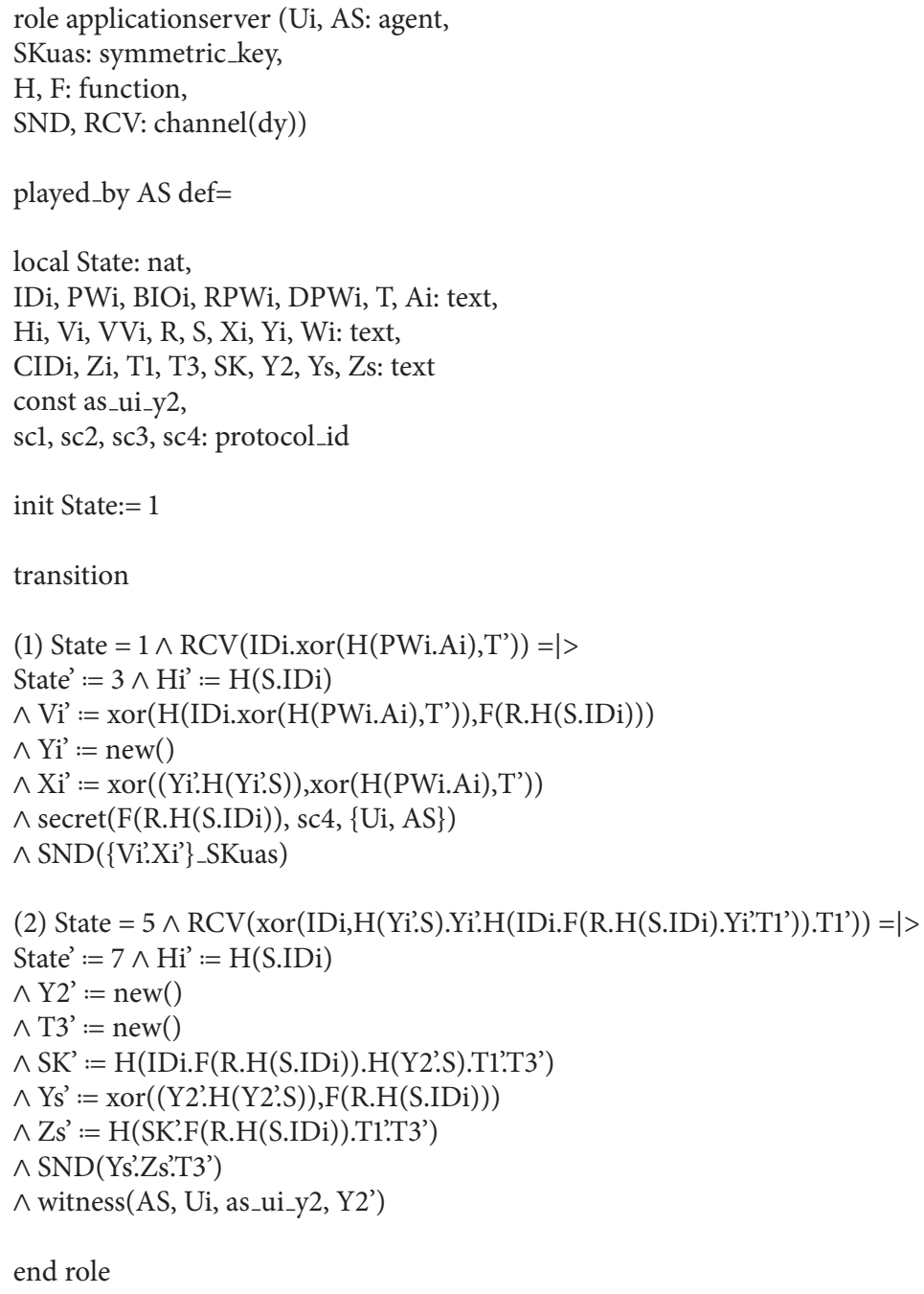

Algorithm 3: Role specification for application server AS.

secret key $s$ of $S$ is known to $\mathscr{E}$. However, $\mathscr{E}$ does not know $T_{r}\left(H_{i}\right)$. Thus, the session key $\lambda=h\left(I D_{i}\left\|T_{r}\left(H_{i}\right)\right\| h\left(y_{i}^{\prime} \|\right.\right.$ s) $\left\|T_{1}\right\| T_{3}$ ) of the improved protocol is still undiscovered to $\mathscr{E}$. Therefore, forward secrecy is retained in the proposed protocol.

\section{Comparison of Functionality and Performance}

This section presents comparisons of the functionality between the improved protocol and related protocols [2328 , and the computational spending between the improved protocol and the other protocols [25-30] is also compared here.

7.1. Functionality Analysis. Table 1 compares the security features provided by the proposed protocol with previous protocols. The results indicate that the proposed protocol is distinctly invulnerable and achieves all of the avoidance requirements.

7.2. Performance Analysis. We demonstrated the computational cost of the improved protocol against previous protocols in terms of the computational cost. According to the simulations obtained in [34], we found that $T_{c} \approx 32.40 \mathrm{~ms}$ and $T_{h} \approx 0.20 \mathrm{~ms}$, respectively, with a system using Pentium IV 3.2 G (CPU) with a $3.0 \mathrm{~GB}$ (RAM). According to [47], the computational cost of the fuzzy extractor technique $T_{f}$ is nearly identical to ECC multiplication. Kilinc and Yanik [48] has gauged the execution time of some cryptographic algorithms by using the Pairing-Based Cryptography Library (version 0.5.12) [49] in the OS: 32-bit Ubuntu 12.04.1, 2.2 G (CPU), and $2.0 \mathrm{G}$ (RAM). They demonstrated that the cost to perform an elliptic curve point multiplication $T_{e}$ is nearly $2.226 \mathrm{~ms}$. In addition, they proved that the cost of a bitwise XOR operation is negligible. In Table 2, we presented the 
TABLE 1: Functionality comparison of the improved protocol with others.

\begin{tabular}{|c|c|c|c|c|c|c|c|}
\hline Property & [23] & {$[24]$} & {$[25]$} & {$[26]$} & [27] & {$[28]$} & The proposed \\
\hline Mutual authentication & $\times$ & $\times$ & $\times$ & $\times$ & $\sqrt{ }$ & $\times$ & $\sqrt{ }$ \\
\hline User anonymity & $x$ & $x$ & $x$ & $x$ & $\sqrt{ }$ & $\sqrt{ }$ & $\sqrt{ }$ \\
\hline Impersonation attack & $x$ & $x$ & $x$ & $x$ & $x$ & $x$ & $\sqrt{ }$ \\
\hline Insider attack & $x$ & $x$ & $\sqrt{ }$ & $\sqrt{ }$ & $\sqrt{ }$ & $x$ & $\sqrt{ }$ \\
\hline DoS attack & $\sqrt{ }$ & $\sqrt{ }$ & $x$ & $\sqrt{ }$ & $\sqrt{ }$ & $\sqrt{ }$ & $\sqrt{ }$ \\
\hline Replay attack & $x$ & $\sqrt{ }$ & $x$ & $\sqrt{ }$ & $\sqrt{ }$ & $\sqrt{ }$ & $\sqrt{ }$ \\
\hline Off-line password guessing attack & $x$ & $\sqrt{ }$ & $x$ & $x$ & $\sqrt{ }$ & $x$ & $\sqrt{ }$ \\
\hline Stolen verifier attack & $x$ & $\sqrt{ }$ & $\sqrt{ }$ & $\sqrt{ }$ & $\sqrt{ }$ & $\sqrt{ }$ & $\sqrt{ }$ \\
\hline Session key attack & $x$ & $x$ & $x$ & $x$ & $x$ & $x$ & $\sqrt{ }$ \\
\hline Provable security & $x$ & $\times$ & $x$ & $x$ & $\sqrt{ }$ & $x$ & $\sqrt{ }$ \\
\hline
\end{tabular}

role session (Ui, AS: agent,

SKuas: symmetric_key,

$\mathrm{H}, \mathrm{F}$ : function)

def $=$

local H1, H2, R1, R2: channel (dy)

composition

user (Ui, AS, SKuas, H, F, H1, R1)

$\wedge$ applicationserver (Ui, AS, SKuas, H, F, H2, R2)

end role

role environment () def $=$

const ui, as: agent,

skuas: symmetric_key,

$\mathrm{h}, \mathrm{f}$ : function,

cidi, yi, zi, tl, ys, zs, t3: text,

as_ui_y2,

sc1, sc2, sc3, sc4: protocol_id

intruder_knowledge $=u i$, as, h, f, cidi, yi, zi, tl, ys, zs, t3

composition

session(ui, as, skuas, $\mathrm{h}, \mathrm{f}$ )

$\wedge \operatorname{session}(i$, as, skuas, $h, f)$

$\wedge$ session(ui, i, skuas, $h, f$ )

end role

goal

secrecy_of sc1, sc2, sc3, sc4

authentication_on as_ui_y2

end goal

environment()

Algorithm 4: Role specification for session, goal, and environment.
$\%$ OFMC

$\%$ Version of 2006/02/13

SUMMARY

SAFE

DETAILS

BOUNDED_NUMBER_OF_SESSIONS

PROTOCOL

/home/span/span/testsuite/results/testrv3.if

GOAL

as_specified

BACKEND

OFMC

COMMENTS

STATISTICS

parseTime: $0.00 \mathrm{~s}$

searchTime: $0.03 \mathrm{~s}$

visiteNodes: 4 nodes

depth: 2 piles

Algorithm 5: The result of simulation using OFMC backends.

computational cost of the improved protocol for each phase and execution time (millisecond) with the related schemes. Compared to Islam et al.s protocol, the improved protocol performs seven further hash functions and two fuzzy-extract operations. However, we reduce four extended chaotic operations. The improved protocol therefore is more effective than Islam et al.s protocol.

\section{Conclusion}

Recently, Islam et al. demonstrated the security vulnerabilities in Lin et al.s protocol and presented an improved authentication protocol using extended chaotic map. Islam 
TABLE 2: Performance comparison of the improved protocol with others.

\begin{tabular}{lccccccc}
\hline & {$[25]$} & {$[29]$} & {$[26]$} & {$[27]$} & {$[28]$} & [30] & The proposed \\
\hline Registration & $3 T_{h}$ & $4 T_{h}$ & $4 T_{h}+T_{c}$ & $6 T_{h}+T_{c}$ & $4 T_{h}$ & $3 T_{h}+T_{c}$ & $7 T_{h}+T_{c}+T_{f}$ \\
Login & $5 T_{h}+T_{c}$ & $3 T_{h}+2 T_{c}$ & $2 T_{h}+2 T_{c}$ & $3 T_{h}+2 T_{c}$ & $5 T_{h}+2 T_{c}$ & $2 T_{h}+3 T_{c}$ & $3 T_{h}+T_{f}$ \\
Verification & $6 T_{h}+5 T_{c}$ & $6 T_{h}+4 T_{c}$ & $6 T_{h}+T_{c}$ & $6 T_{h}+T_{c}$ & $7 T_{h}+4 T_{c}$ & $6 T_{h}+2 T_{c}$ & $8 T_{h}+1 T_{c}$ \\
Total & $14 T_{h}+6 T_{c}$ & $13 T_{h}+6 T_{c}$ & $14 T_{h}+4 T_{c}$ & $15 T_{h}+4 T_{c}$ & $16 T_{h}+6 T_{c}$ & $11 T_{h}+6 T_{c}$ & $18 T_{h}+2 T_{c}+2 T_{f}$ \\
Time $(\mathrm{ms})$ & $\approx 197.2$ & $\approx 197.0$ & $\approx 132.4$ & $\approx 132.6$ & $\approx 197.6$ & $\approx 196.6$ & $\approx 72.9$ \\
\hline
\end{tabular}

et al. also asserted that their authentication protocol is more secure than Lin et al's protocol and that it guarantees user anonymity. However, Islam et al.s protocol is still insecure against some types of attacks, such as on-line identity guessing and user impersonation. To overcome these security weaknesses, in the current paper, we suggest an improved user authentication protocol using a fuzzy extractor that preserves the advantages of Islam et al's protocol and contributes to inclusive security properties. The formal and informal analyses of this work clarify why the improved protocol is more efficient and secure.

\section{Notations}

$\begin{array}{ll}U_{i}: & \text { Mobile user } \\ \mathrm{MD}_{i}: & \text { Mobile device of user } \\ \mathrm{ID}_{i}: & \text { Identity of user } \\ \mathrm{PW}_{i}: & \text { Password of user } \\ \mathrm{BIO}_{i}: & \text { Biometrics of user } \\ S: & \text { Remote server } \\ x: & \text { Real number chosen set }[-1,1] \\ T_{k}(x): & \text { Chebyshev polynomial of degree } k \\ s: & \text { Master secret key of server } S \\ r: & \text { Positive random integer generated server } S \\ h(\cdot): & \text { Cryptographic hash function } \\ 9 \alpha_{i}, \beta_{i}: & U_{i} \text { 's nearly random binary and auxiliary } \\ \lambda: & \text { binary strings } \\ \lambda: & \text { Session key } \\ T: & \text { Timestamp } \\ \|: & \text { Concatenation operator } \\ \oplus: & \text { Bitwise XOR operator. }\end{array}$

\section{Conflicts of Interest}

The authors declare that they have no conflicts of interest.

\section{Acknowledgments}

This research was supported by Basic Science Research Program through the National Research Foundation of Korea (NRF) funded by the Ministry of Education (NRF-20100020210).

\section{References}

[1] N. Park, H. W. Kim, S. Kim, and D. Won, "Open location-based service using secure middleware infrastructure in web services," in Proceedings of the International Conference on Computational
Science and Its Applications - ICCSA 2005, pp. 1146-1155, sgp, May 2005.

[2] L. Lamport, "Password authentication with insecure communication," Communications of the ACM, vol. 24, no. 11, pp. 770772,1981

[3] M. Kumar, "On the weaknesses and improvements of an efficient password based remote user authentication scheme using smart cards," IACR Cryptology ePrint Archive, pp. 163-174, 2004.

[4] H. Lin, "Efficient mobile dynamic ID authentication and key agreement scheme without trusted servers," International Journal of Communication Systems, vol. 30, no. 1, Article ID e2818, 2017.

[5] M. Khan and J. Zhang, "Improving the security of "a flexible biometrics remote user authentication scheme"," Computer Standards and Interfaces, vol. 29, no. 1, pp. 82-85, 2007.

[6] W. Jeon, J. Kim, J. Nam, Y. Lee, and D. Won, "An enhanced secure authentication scheme with anonymity for wireless environments," IEICE Transactions on Communications, vol. 95, no. 7, pp. 2505-2508, 2012.

[7] D. He, N. Kumar, M. K. Khan, and J.-H. Lee, "Anonymous twofactor authentication for consumer roaming service in global mobility networks," IEEE Transactions on Consumer Electronics, vol. 59, no. 4, pp. 811-817, 2013.

[8] D. Mishra, A. Das, and S. Mukhopadhyay, "A secure user anonymity-preserving biometric-based multi-server authenticated key agreement scheme using smart cards," Expert Systems with Applications, vol. 41, no. 18, pp. 8129-8143, 2014.

[9] R. Amin, S. Islam, G. Biswas, M. Khan, and N. Kumar, "A robust and anonymous patient monitoring system using wireless medical sensor networks," Future Generation Computer Systems, 2015.

[10] R. Amin, R. Sherratt, D. Giri, S. Islam, and M. Khan, "A software agent enabled biometric security algorithm for secure file access in consumer storage devices," IEEE Transactions on Consumer Electronics, vol. 63, no. 1, pp. 53-61, 2017.

[11] P. Mohit, R. Amin, and G. Biswas, "Design of authentication protocol for wireless sensor network-based smart vehicular system," Vehicular Communications, vol. 9, pp. 64-71, 2017.

[12] A. Chaturvedi, D. Mishra, S. Jangirala, and S. Mukhopadhyay, "A privacy preserving biometric-based three-factor remote user authenticated key agreement scheme," Journal of Information Security and Applications, vol. 32, pp. 15-26, 2017.

[13] D. Mishra, S. Kumari, M. Khan, and S. Mukhopadhyay, "An anonymous biometric-based remote user-authenticated key agreement scheme for multimedia systems," International Journal of Communication Systems, vol. 30, no. 1, Article ID e2946, 2017.

[14] S. Park, S. Kim, and D. Won, "ID-based group signature," Electronics Letters, vol. 33, no. 19, pp. 1616-1617, 1997. 
[15] R. Amin and G. Biswas, "An Improved RSA Based User Authentication and Session Key Agreement Protocol Usable in TMIS," Journal of Medical Systems, vol. 39, no. 8, article no. 79, 2015.

[16] J. Nam, M. Kim, J. Paik, Y. Lee, and D. Won, "A provablysecure ECC-based authentication scheme for wireless sensor networks," Sensors, vol. 14, no. 11, pp. 21023-21044, 2014.

[17] R. Amin, S. Islam, G. Biswas, M. Khan, and N. Kumar, "An Efficient and Practical Smart Card Based Anonymity Preserving User Authentication Scheme for TMIS using Elliptic Curve Cryptography," Journal of Medical Systems, vol. 39, no. 11, article no. 180, 2015.

[18] C. Chen, D. He, S. Chan, J. Bu, Y. Gao, and R. Fan, "Lightweight and provably secure user authentication with anonymity for the global mobility network," International Journal of Communication Systems, vol. 24, no. 3, pp. 347-362, 2011.

[19] H. Debiao, C. Jianhua, and Z. Rui, "A more secure authentication scheme for telecare medicine information systems," Journal of Medical Systems, vol. 36, no. 3, pp. 1989-1995, 2012.

[20] S. Wu, Y. Zhu, and Q. Pu, "Robust smart-cards-based user authentication scheme with user anonymity," Security and Communication Networks, vol. 5, no. 2, pp. 236-248, 2012.

[21] P. Gong, P. Li, and W. Shi, "A secure chaotic maps-based key agreement protocol without using smart cards," Nonlinear Dynamics. An International Journal of Nonlinear Dynamics and Chaos in Engineering Systems, vol. 70, no. 4, pp. 2401-2406, 2012.

[22] J. Moon, Y. Choi, J. Kim, and D. Won, "An Improvement of Robust and Efficient Biometrics Based Password Authentication Scheme for Telecare Medicine Information Systems Using Extended Chaotic Maps," Journal of Medical Systems, vol. 40, no. 3, article no. 70, pp. 1-11, 2016.

[23] D. Xiao, X. Liao, and S. Deng, "A novel key agreement protocol based on chaotic maps," Information Sciences. An International Journal, vol. 177, no. 4, pp. 1136-1142, 2007.

[24] S. Han, H. Tseng, R. Jan, and W. Yang, "A chaotic mapsbased key agreement protocol that preserves user anonymity," in Proceedings of the IEEE International Conference on Communications (ICCâ09, pp. 1-6, Dresden, Germany, 2009.

[25] C. Lee, C. Chen, C. Wu, and S. Huang, "An extended chaotic maps-based key agreement protocol with user anonymity," Nonlinear Dynamics. An International Journal of Nonlinear Dynamics and Chaos in Engineering Systems, vol. 69, no. 1-2, pp. 79-87, 2012.

[26] H. Lin, "Chaotic map based mobile dynamic ID authenticated key agreement scheme," Wireless Personal Communications, vol. 78, no. 2, pp. 1487-1494, 2014.

[27] S. Islam, M. Obaidat, and R. Amin, "An anonymous and provably secure authentication scheme for mobile user," International Journal of Communication Systems, vol. 29, no. 9, pp. 1529-1544, 2016.

[28] C. Lee and C. Hsu, "A secure biometric-based remote user authentication with key agreement scheme using extended chaotic maps," Nonlinear Dynamics. An International Journal of Nonlinear Dynamics and Chaos in Engineering Systems, vol. 71, no. 1-2, pp. 200-211, 2013.

[29] D. He, Y. Chen, and J. Chen, "Cryptanalysis and improvement of an extended chaotic maps-based key agreement protocol," Nonlinear Dynamics. An International Journal of Nonlinear Dynamics and Chaos in Engineering Systems, vol. 69, no. 3, pp. 1149-1157, 2012.

[30] D. Guo, Q. Wen, W. Li, H. Zhang, and Z. Jin, "Analysis and Improvement of 'Chaotic Map Based Mobile Dynamic ID
Authenticated Key Agreement Scheme"' Wireless Personal Communications, vol. 83, no. 1, pp. 35-48, 2015.

[31] S. Han, "Security of a key agreement protocol based on chaotic maps," Chaos, Solitons \& Fractals, vol. 38, no. 3, pp. 764-768, 2008.

[32] Y. Niu and X. Wang, "An anonymous key agreement protocol based on chaotic maps," Communications in Nonlinear Science and Numerical Simulation, vol. 16, no. 4, pp. 1986-1992, 2011.

[33] E. Yoon, "Efficiency and security problems of anonymous key agreement protocol based on chaotic maps," Communications in Nonlinear Science and Numerical Simulation, vol. 17, no. 7, pp. 2735-2740, 2012.

[34] K. Xue and P. Hong, "Security improvement of an anonymous key agreement protocol based on chaotic maps," Communications in Nonlinear Science and Numerical Simulation, vol. 17, no. 7, pp. 2969-2977, 2012.

[35] Z. Tan, "A chaotic maps-based authenticated key agreement protocol with strong anonymity," Nonlinear Dynamics. An International Journal of Nonlinear Dynamics and Chaos in Engineering Systems, vol. 72, no. 1-2, pp. 311-320, 2013.

[36] C. Li, C. Lee, and C. Weng, "An extended chaotic maps based user authentication and privacy preserving scheme against DoS attacks in pervasive and ubiquitous computing environments," Nonlinear Dynamics. An International Journal of Nonlinear Dynamics and Chaos in Engineering Systems, vol. 74, no. 4, pp. 1133-1143, 2013.

[37] D. Dolev and A. Yao, "On the security of public key protocols," Institute of Electrical and Electronics Engineers. Transactions on Information Theory, vol. 29, no. 2, pp. 198-208, 1983.

[38] J. Moon, Y. Choi, J. Jung, and D. Won, "An improvement of robust biometrics-based authentication and key agreement scheme for multi-server environments using smart cards," PLoS ONE, vol. 10, no. 12, Article ID e0145263, 2015.

[39] Y. Dodis, B. Kanukurthi, J. Katz, and A. Smith, "Robust fuzzy extractors and authenticated key agreement from close secrets," IEEE Transactions on Information Theory, vol. 58, no. 9, pp. 6207-6222, 2012.

[40] A. Das, "A secure and effective biometric-based user authentication scheme for wireless sensor networks using smart card and fuzzy extractor," International Journal of Communication Systems, vol. 30, no. 1, Article ID e2933, 2017.

[41] C. Wang, X. Zhang, and Z. Zheng, "Cryptanalysis and improvement of a biometric-based user authentication scheme for wireless sensor networks using smart card and fuzzy extractor," in PLoS One, vol. 11, pp. 25-25, 2016.

[42] Y. Dodis, L. Reyzin, and A. Smith, "Fuzzy extractors: how to generate strong keys from biometrics and other noisy data," in Advances in cryptology-EUROCRYPT 2004, vol. 3027 of Lecture Notes in Comput. Sci., pp. 523-540, Springer, Berlin, 2004.

[43] P. Bergamo, P. D’Arco, A. De Santis, and L. Kocarev, "Security of public-key cryptosystems based on Chebyshev polynomials," IEEE Transactions on Circuits and Systems. I. Regular Papers, vol. 52, no. 7, pp. 1382-1393, 2005.

[44] A. Das, "A secure and effective user authentication and privacy preserving protocol with smart cards for wireless communication," in Networking Science, vol. 2, pp. 12-27, 2, 2013.

[45] Y. Lu, L. Li, X. Yang, and Y. Yang, "Robust biometrics based authentication and key agreement scheme for multi-server environments using smart cards," PLoS ONE, vol. 10, no. 5, Article ID 0126323, 2015. 
[46] von Oheimb D. The high-level protocol specification language hlpsl developed in the eu project avispa. In Proceedings of the Applied Semantics 2005 Workshop, Frauenchiemsee, Germany, 12-15 September 2005; pp. 1-17.

[47] M. Wazid, A. K. Das, S. Kumari, X. Li, and F. Wu, "Design of an efficient and provably secure anonymity preserving three-factor user authentication and key agreement scheme for TMIS," Security and Communication Networks, vol. 9, no. 13, pp. 19832001, 2016.

[48] H. Kilinc and T. Yanik, "A survey of SIP authentication and key agreement schemes," IEEE Communications Surveys and Tutorials, vol. 16, no. 2, pp. 1005-1023, 2014.

[49] Lynn B. Pairing-based cryptography library, available at http:// crypto.stanford.edu/pbc/. 


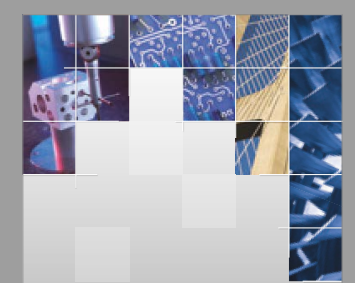

\section{Enfincering}
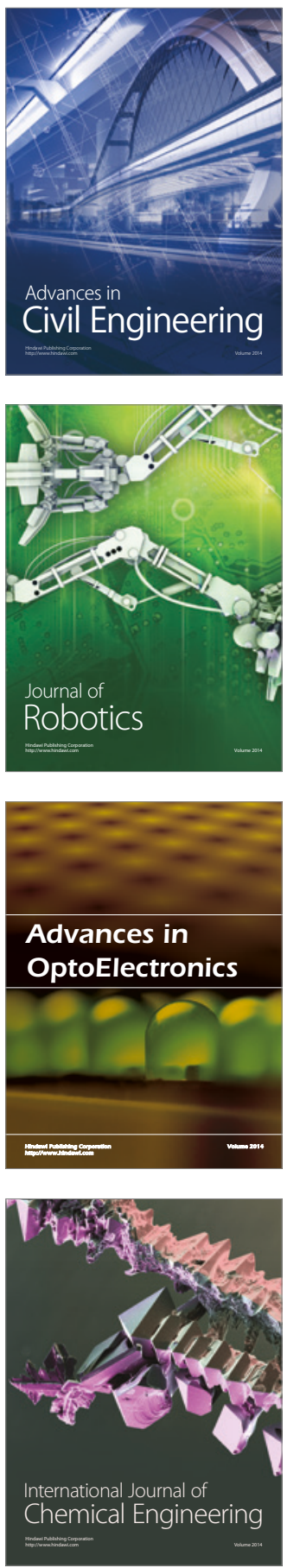

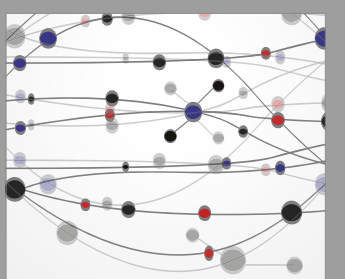

The Scientific World Journal

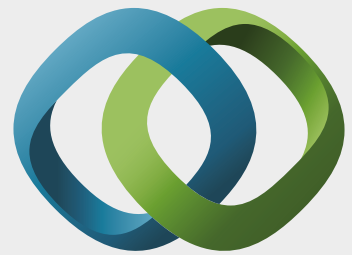

\section{Hindawi}

Submit your manuscripts at

https://www.hindawi.com
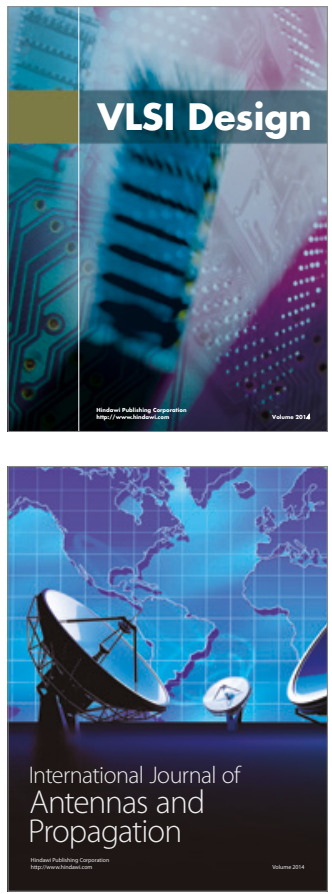

\section{Rotating}

Machinery
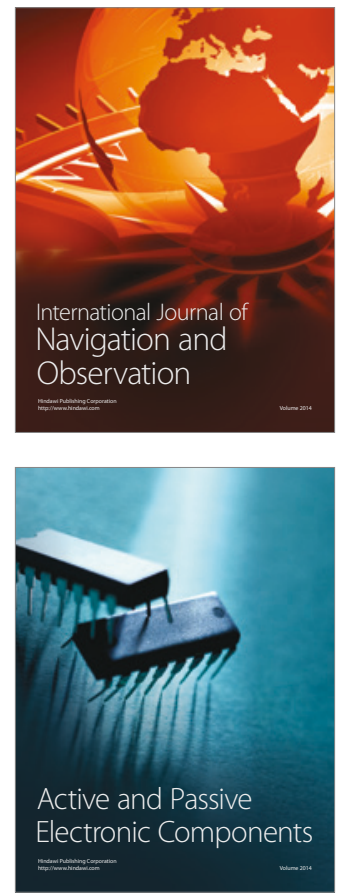
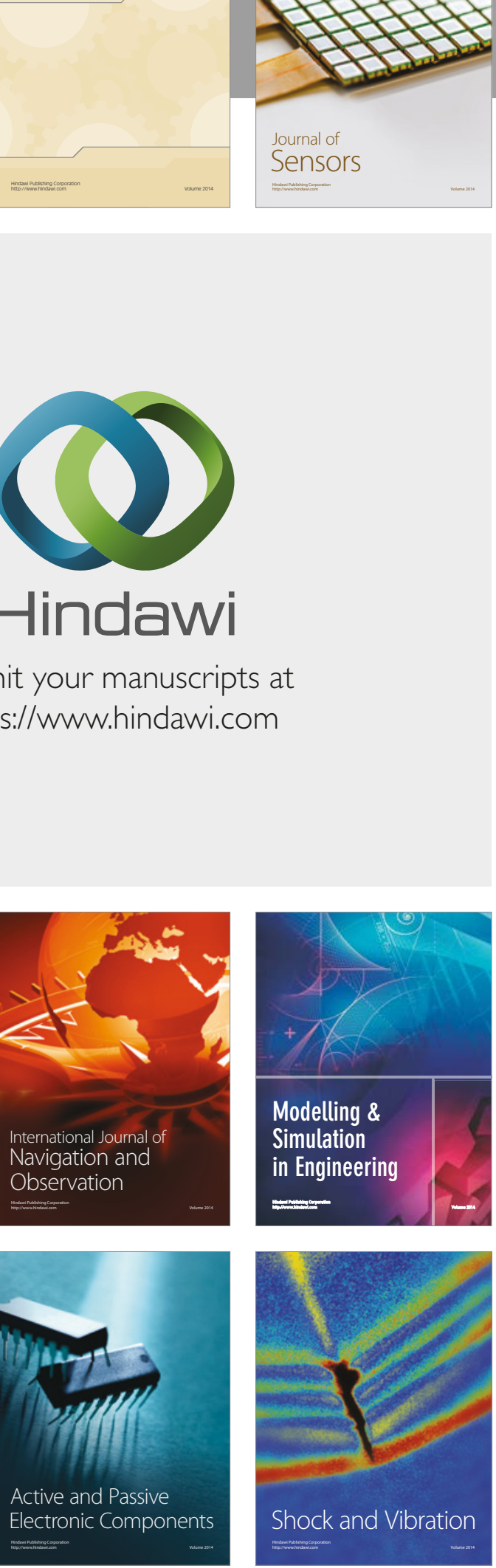
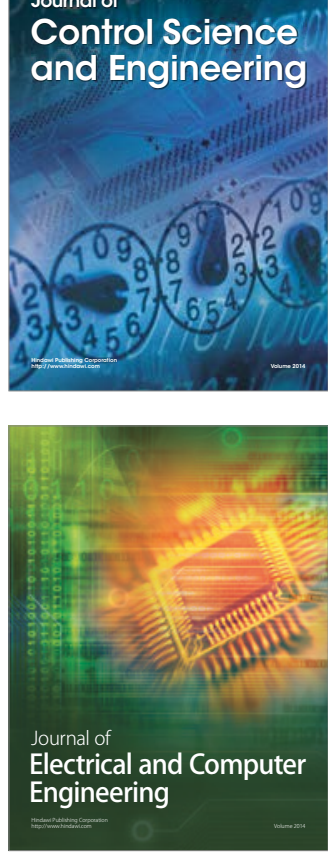

Distributed

Journal of

Control Science

and Engineering
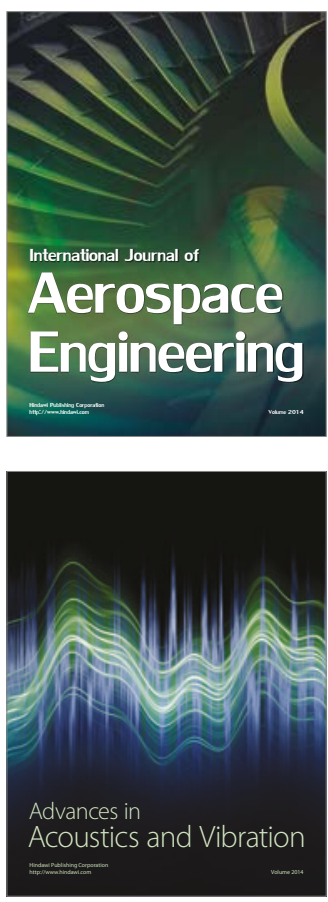

Sensor Networks 